\title{
ESTIMATIVA DO ESCOAMENTO SUPERFICIAL EM UMA BACIA HIDROGRÁFICA COM BASE EM MODELAGEM DINÂMICA E DISTRIBUÍDA ${ }^{(1)}$
}

\author{
Samuel Beskow ${ }^{(2)}$, Carlos Rogério de Mello ${ }^{(3)}$, Gilberto Coelho ${ }^{(4)}$, Antônio \\ Marciano da Silva ${ }^{(3)}$ \& Marcelo Ribeiro Viola ${ }^{(5)}$
}

\begin{abstract}
RESUMO
A estimativa do escoamento superficial em bacias hidrográficas é de suma importância para conservação dos recursos naturais; entretanto, esse é um processo complexo e dinâmico, principalmente no contexto de sua variabilidade espacial. Dessa forma, torna-se adequada a aplicação dos Sistemas de Informações Geográficas (SIG) usando pequenas células de informação, pois assim é possível considerar o comportamento espacial das variáveis associadas à origem do escoamento superficial. Este trabalho teve como objetivo implementar os modelos hidrológicos Curva Número (CN-SCS) e Curva-Número Modificado (CN-MMS), com base na linguagem de programação do SIG PCRaster e em uma base de dados reduzida, de forma distribuída e dinâmica, com o intuito de estimar as lâminas de escoamento superficial geradas numa bacia hidrográfica de Latossolos, localizada no município de Nazareno, região dos Campos das Vertentes, Minas Gerais. Para aplicação do modelo CN-SCS foi preciso desenvolver um mapa com valores de CN no formato do PCRaster, enquanto para o modelo CN-MMS foram necessários os seguintes mapas: umidade volumétrica de saturação do solo, umidade volumétrica inicial do solo e profundidade de solo. Para simulação e avaliação de ambos os modelos, foram aplicados 18 eventos de chuva natural que provocaram escoamento superficial, durante o ano hidrológico 2004-2005, e suas respectivas lâminas de escoamento observadas. A análise do desempenho dos modelos foi feita aplicandose análise de sensibilidade baseada no erro médio e na Raiz do Erro Quadrático (REQ). Tendo-se como referência essas estatísticas de precisão, pôde-se constatar que o modelo CN-MMS apresentou melhor calibração quando comparado ao modelo CN-SCS, devido à consideração direta da umidade inicial do solo. Contudo, a
\end{abstract}

\footnotetext{
(1) Pesquisa financiada pelo CNPQ (Processo 472274/2007-4), FAPEMIG (CAG 1617/06) e CEMIG/ANEEL (P\&D 176). Recebido para publicação em junho de 2008 e aprovado em dezembro de 2008.

(2) Doutorando do Programa de Pós-graduação em Eng. Agrícola da UFLA e Visiting Scholar - Purdue University. Bolsista do CNPQ. E-mail: sbeskow@purdue.edu

${ }^{(3)}$ Professor do Departamento de Engenharia da Universidade Federal de Lavras - DEG/UFLA. Caixa Postal 37, CEP 37200-000, Lavras (MG). Bolsistas do CNPQ. E-mail: crmello@ufla.br; marciano@ufla.br

(4) Pesquisador, bolsista PRODOC/CAPES, DEG/UFLA. E-mail: coelho@ufla.br

${ }^{(5)}$ Doutorando do Programa de Pós-graduação em Eng. Agrícola, DEG/UFLA. E-mail: mrviola@eagricola.ufla.br
} 
estruturação dos modelos no SIG PCRaster possibilitou o desenvolvimento de uma ferramenta computacional eficaz e útil para simulação do escoamento superficial, visto que propicia estruturação de rotinas computacionais considerando os problemas associados à variabilidade espacial dos dados de entrada dos modelos.

Termos de indexação: conservação de solo e água, simulação, SIG, PCRaster.

\title{
SUMMARY: SURFACE RUNOFF IN A WATERSHED ESTIMATED BY DYNAMIC AND DISTRIBUTED MODELING
}

\begin{abstract}
Knowledge on the surface runoff in watersheds is very important for a good conservation of natural resources. However, surface runoff is a complex and dynamic process, especially in the context of spatial variability. An application of the Geographical Information System (GIS) tools in small grid cells is therefore adequate. This way, it is possible to consider the spatial behavior of variables associated to the origin of surface runoff. This study aimed to program the Hydrological Models SCS-CN (SCS-Curve Number) and CN - MMS (CNModified) based on the PC Raster Programming Language and a reduced dataset, in distributed and dynamic approaches, to estimate the direct surface runoff in a watershed of Oxissols, in the county of Nazareno, region of Campos das Vertentes, Minas Gerais State. SCS-CN model was applied structuring a CN-map linked to the soil infiltration capacity in the PCRaster GIS format. For the CN-MMS model, we used other maps: saturated volumetric soil moisture content, initial volumetric soil moisture content and soil depth. For the simulation and evaluation of both models, 18 rainfall events that produced surface runoff in the hydrologic year 2004-2005 were used, and their respective surface runoff depths observed. The model performance was evaluated by sensitivity analyses, based on the mean error and Root Square Error. Based on these accuracy statistics, the calibration of the CN-MMS model was better than in the original SCS-CN model, since the initial soil moisture was taken into account. Nevertheless, the adjustment of the models in the SIG PCRaster allowed the development of an effective and useful computer tool to simulate surface runoff, due to the possibility of establishing computer routines considering the problems elated to the spatial variability of data entries of the model.
\end{abstract}

Index terms: soil and water conservation, simulation, GIS, PCRaster.

\section{INTRODUÇÃO}

Em decorrência do crescimento da população, existe a necessidade do aumento de uso de terras cultiváveis, podendo conduzir à degradação do solo e dos recursos hídricos. Dessa forma, é muito importante o desenvolvimento de métodos ligados à gestão dos recursos naturais solo e água para minimizar os impactos sobre estes.

De acordo com Pedrosa \& Câmara (2007), a técnica de Geoprocessamento tem utilizado a representação de fenômenos espaciais no computador de forma estática, visto que a principal abstração utilizada em Sistemas de Informações Geográficas (SIG) é o mapa. Contudo, alguns fenômenos espaciais, como o escoamento de água da chuva, são inerentemente dinâmicos e as representações estáticas normalmente utilizadas em SIG não o capturam de forma adequada.

Conforme Bacic et al. (2008), trabalhando com o modelo AgNPS, a simulação dinâmica e distribuída do comportamento ambiental em bacias hidrográficas pode auxiliar no planejamento e gerenciamento de bacias de drenagem carentes de dados hidrológicos e sujeitas a constantes mudanças no uso do solo.

Entretanto, a simulação hidrológica do escoamento superficial em bacias hidrográficas é extremamente complexa; assim, para utilização de modelos computacionais aplicados à sua simulação, estes devem apresentar algumas características desejáveis (Mello et al., 2008), como serem baseados no processo físico, no evento e na distribuição espacial das variáveis associadas ao fenômeno. Com base no processo físico significa que o modelo deve incorporar os principais agentes físicos envolvidos na geração do escoamento superficial e erosão; no evento, que os processos são mensurados no modelo com base em incrementos de tempo, desde seu início até o final do evento; e na distribuição espacial das variáveis, que os processos físicos em questão são observados com base em células hidrologicamente homogêneas, ou seja, a bacia hidrográfica é dividida em células de tamanho uniforme com características pedológicas e de uso do solo semelhantes, reduzindo problemas associados à variabilidade espacial. 
Segundo De Roo \& Jetten (1999), a principal razão para se usar SIG é que o processo de escoamento superficial varia espacialmente e, assim, células de informação devem ser usadas para que a variação espacial seja levada em consideração. Conforme esses autores, outro aspecto importante é a necessidade de uma grande quantidade de dados para o extenso número de células requeridas, dados estes que não são facilmente obtidos e gerenciados sem intermédio de um SIG.

O PCRaster consiste de um sistema de modelagem dinâmica para modelos distribuídos de simulação hidrossedimentológica (van Deursen, 1995; Wesseling et al., 1996), fornecendo um excelente ambiente para modelos distribuídos, pois embute uma linguagem de modelagem dinâmica ambiental em um Sistema de Informações Geográficas. A linguagem de modelagem dinâmica é um instrumento poderoso para construção de modelos ambientais, a qual pode ser regulada para o problema em estudo.

Diante do exposto, este trabalho teve como objetivos aplicar os modelos hidrológicos Curva-Número (Soil Conservation Service, 1971) e Curva-Número Modificado (CN-MMS - Mishra et al., 2003) a uma bacia hidrográfica de Latossolos, utilizando uma ferramenta baseada nos Sistemas de Informações Geográficas (SIG), em linguagem de programação do SIG PCRaster, simulando o escoamento superficial direto e levando em consideração a distribuição espacial e temporal das variáveis de entrada que influenciam o comportamento do fenômeno.

\section{MATERIAL E MÉTODOS}

\section{Caracterização da área de estudo}

A área de estudo compreende a bacia hidrográfica do Ribeirão Marcela, localizada no município de Nazareno, região fisiográfica dos Campos das Vertentes, Minas Gerais, com área aproximada de 477 ha e declividade média de $13,64 \%$, drenando para o reservatório da UHE de Itutinga/Camargos. Essa bacia hidrográfica é representativa do domínio dos Latossolos nessa região, os quais ocupam $70 \%$ da bacia e apresentam alta capacidade de recarga de aquíferos, de acordo com estudos pedológicos e hidrogeológicos realizados previamente por Araújo (2006). No que se refere ao uso atual do solo, a bacia hidrográfica do Ribeirão Marcela apresenta pastagens, milho, café e mata (Figura 1). Os dados hidrológicos trabalhados correspondem a 18 eventos chuva-vazão monitorados na referida bacia hidrográfica durante o ano hidrológico 2004-2005, ou seja, foram selecionados eventos de chuva natural que provocaram escoamento superficial direto, os quais foram extraídos, conforme Tucci (2005), a partir da análise do hidrograma de escoamento monitorado na seção de controle da bacia. Ressalta-se que os eventos selecionados para simulação provocaram hidrogramas de escoamento de boa qualidade, com duração próxima ao tempo de concentração da bacia, estimado entre 45 e $60 \mathrm{~min}$, possibilitando a identificação e separação precisa do escoamento superficial direto do subterrâneo, algo indispensável para avaliação do desempenho dos modelos.
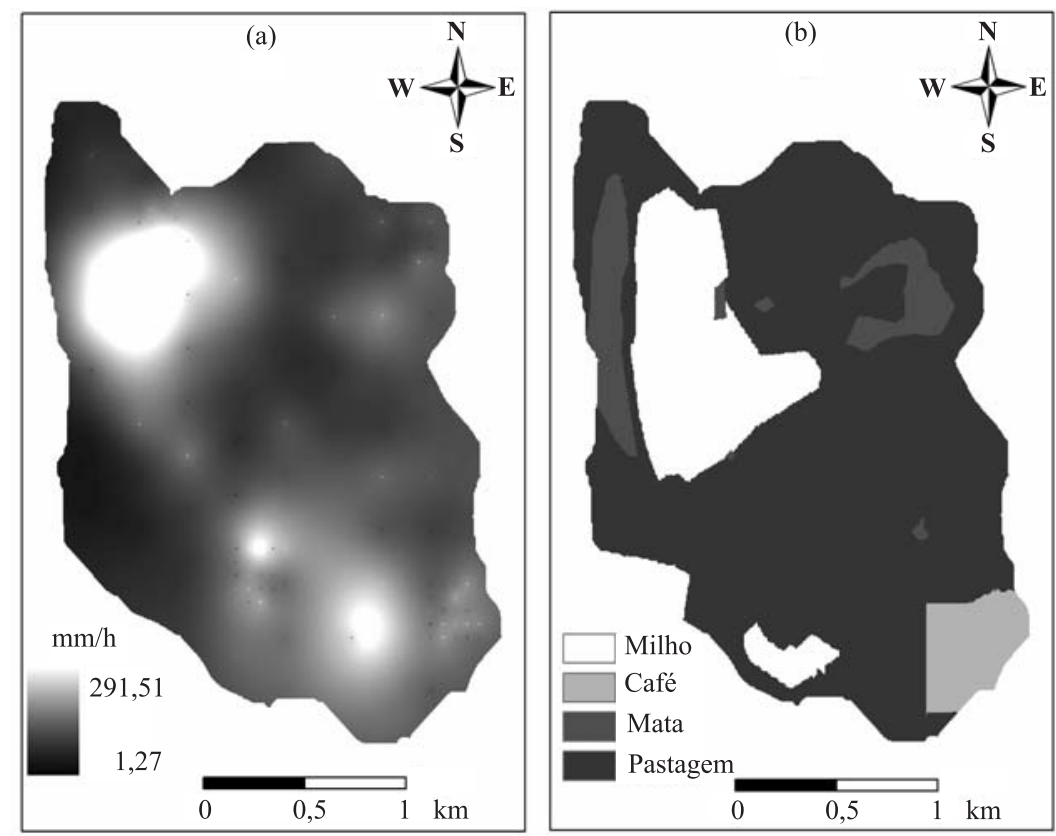

Figura 1. Mapas de taxa de infiltração (a) e uso atual do solo (b) aplicados ao SIG PCRaster para simulação com os modelos CN-SCS e CN-MMS. 
No quadro 1 apresentam-se os eventos chuva-vazão observados na bacia e aplicados ao estudo.

\section{Modelo curva número (CN-SCS)}

O modelo hidrológico Curva-Número (Soil Conservation Service, 1971) é bastante conhecido e difundido na conservação do solo e da água, sendo usado para estimar o escoamento superficial direto gerado por eventos de precipitação pluvail individuais, bem como em modelos de simulação hidrossedimentológicos (Hrissanthou, 2002; Notter et al., 2007; von Stackelberg et al., 2007; Mello et al., 2008);.

De acordo com van Deursen (1995), a equação tradicional desse modelo pode ser reescrita considerando a distribuição do excesso de chuva no tempo, utilizando as seguintes equações:

$$
\begin{gathered}
\mathrm{S}=\frac{25.400}{\mathrm{CN}}-254 \\
\mathrm{Ia}=\lambda . \mathrm{S} \\
\mathrm{Fa}=\frac{\mathrm{S}(\mathrm{P}-\mathrm{Ia})}{(\mathrm{P}-\mathrm{Ia}-\mathrm{S})} \\
\mathrm{D}_{\mathrm{s}}=\mathrm{P}-\mathrm{Fa}
\end{gathered}
$$

em que S é o potencial máximo de infiltração ( $\mathrm{mm}$ )

\begin{tabular}{|c|c|c|c|c|c|}
\hline Evento & Data & $\mathbf{P}^{(1)}$ & $\mathrm{P} 5^{(2)}$ & $\mathbf{E S}^{(3)}$ & $\mathrm{C}^{(4)}$ \\
\hline & & & $\mathrm{mm}$ & & $\%$ \\
\hline 1 & $31 / 01 / 2004$ & 37,00 & 0,00 & 0,660 & 1,78 \\
\hline 2 & 08/02/2004 & 6,00 & 7,00 & 0,104 & 1,74 \\
\hline 3 & $10 / 02 / 2004$ & 10,00 & 13,00 & 0,209 & 2,09 \\
\hline 4 & $14 / 02 / 2004$ & 36,00 & 0,00 & 0,352 & 0,98 \\
\hline 5 & $20 / 02 / 2004$ & 15,00 & 11,00 & 0,095 & 0,63 \\
\hline 6 & $21 / 02 / 2004$ & 17,00 & 22,00 & 0,572 & 3,36 \\
\hline 7 & $23 / 02 / 2004$ & 16,00 & 62,00 & 0,411 & 2,57 \\
\hline 8 & $23 / 02 / 2004$ & 6,00 & 100,00 & 0,378 & 6,30 \\
\hline 9 & $24 / 02 / 2004$ & 37,00 & 106,00 & 0,414 & 1,12 \\
\hline 10 & $19 / 03 / 2004$ & 25,20 & 4,40 & 0,454 & 1,80 \\
\hline 11 & $04 / 04 / 2004$ & 11,20 & 0,00 & 0,178 & 1,59 \\
\hline 12 & 04/04/2004 & 45,40 & 11,20 & 0,629 & 1,39 \\
\hline 13 & 05/04/2004 & 30,10 & 56,60 & 0,375 & 1,25 \\
\hline 14 & 07/04/2004 & 29,50 & 86,70 & 0,319 & 1,08 \\
\hline 15 & $12 / 04 / 2004$ & 22,30 & 12,10 & 0,400 & 1,79 \\
\hline 16 & $26 / 05 / 2004$ & 6,90 & 0,00 & 0,120 & 1,74 \\
\hline 17 & $31 / 05 / 2004$ & 23,90 & 0,60 & 0,462 & 1,93 \\
\hline 18 & $19 / 07 / 2004$ & 12,60 & 0,00 & 0,061 & 0,48 \\
\hline
\end{tabular}
após o início do escoamento superficial; $\mathrm{CN}$, o

Quadro 1. Dados referentes aos eventos de chuvavazão usados na simulação

$\overline{(1)}$ Precipitação pluvial total do evento de chuva. ${ }^{(2)}$ Precipitação pluvial total de cinco dias antecedentes ao evento de chuva considerado. ${ }^{(3)}$ Lâminas de água decorrente do escoamento superficial direto. ${ }^{(4)}$ Coeficiente que expressa a relação entre o escoamento superficial direto e a precipitação pluvial total do evento. parâmetro Curva-Número; Ia, a abstração inicial $(\mathrm{mm}) ; \lambda$, o coeficiente de abstração inicial; $\mathrm{Fa}$, as abstrações acumuladas (mm); $\mathrm{P}$, a precipitação pluvial acumulada $(\mathrm{mm})$; e $\mathrm{D}_{\mathrm{s}}$, o escoamento superficial acumulado $(\mathrm{mm})$. O tipo de solo e a condição de cobertura e de umidade antecedente da bacia de drenagem influenciam os valores de CN (van Deursen, 1995).

\section{Modelo CN-Modificado (CN-MMS)}

No modelo tradicional CN-SCS, a umidade do solo é relacionada com a precipitação pluvial de cinco dias antecedentes, dividindo-se nas condições AMC I (solo seco), AMC II (solo próximo à capacidade de campo) e AMC III (solo muito úmido). Além disso, o coeficiente de abstração inicial é constante ( $\lambda$ ), o qual depende especialmente das condições climáticas, sendo a hipótese mais ambígua, requerendo refinamento (Mello et al., 2007). A partir disso, Mishra et al. (2003) desenvolveram o modelo Mishra-Singh Modificado (CN-MMS), incorporando a umidade antecedente e a precipitação pluvial acumulada de cinco dias anteriores, bem como a variação nos valores de $\lambda$. Nesse modelo, o escoamento superficial é computado pela seguinte sequência de equações:

$$
\begin{gathered}
\mathrm{S}=\left(\theta_{\mathrm{s}}-\theta_{0}\right) \cdot \mathrm{h} \\
\mathrm{M}=0,5\left[-(1+\lambda) \mathrm{S}+\sqrt{(1-\lambda)^{2} \mathrm{~S}^{2}+4 \mathrm{P}_{5} \mathrm{~S}}\right] \\
\mathrm{Ia}=\frac{\lambda \mathrm{S}^{2}}{\mathrm{~S}+\mathrm{M}} \\
\mathrm{D}_{\mathrm{s}}=\frac{(\mathrm{P}-\mathrm{Ia})(\mathrm{P}-\mathrm{Ia}+\mathrm{M})}{\mathrm{P}-\mathrm{Ia}+\mathrm{M}+\mathrm{S}}
\end{gathered}
$$

em que $\theta_{\mathrm{S}}$ é a umidade de saturação do solo $\left(\mathrm{m}^{3} \mathrm{~m}^{-3}\right)$; $\theta_{\mathrm{o}}$, a umidade inicial do solo $\left(\mathrm{m}^{3} \mathrm{~m}^{-3}\right)$; h, a profundidade considerada na simulação $(\mathrm{mm}) ; \mathrm{M}$, um parâmetro associado à umidade antecedente $(\mathrm{mm}) ; \mathrm{e} \mathrm{P}_{5}$, a precipitação pluvial de cinco dias antecedentes (mm). Os demais parâmetros foram definidos anteriormente.

\section{Estruturação dos modelos CN-SCS e CN-MMS em PCRaster e base de dados}

Os modelos CN-SCS e CN-MMS foram implementados na linguagem de programação do SIG PCRaster de acordo com as respectivas equações. O PCRaster é um sistema de modelagem dinâmica para modelos distribuídos de simulação, constituindo um SIG estruturado em um conjunto de ferramentas computacionais para armazenamento, manipulação, análise e acesso de informações geográficas. Possui ainda função SIG em formato Raster e um conjunto de ferramentas para visualização.

O conceito central do PCRaster é a discretização da área no espaço, resultando em células de informação (Jetten, 2003). Cada célula pode ser tratada como um conjunto de atributos que definem suas propriedades hidrológicas, mas uma célula pode receber e transmitir 
informações para células vizinhas. A base de dados do PCRaster suporta quatro tipos de dados: mapas no formato Raster, dados do tipo pontual, tabelas (para relação entre mapas Raster) e séries temporais (para representação dos dados climáticos e hidrológicos variando no tempo).

O modelo digital de elevação (MDE) foi obtido a partir das cartas topográficas de Itutinga $\left(n^{\circ} 26434\right)$ e Nazareno ( $\left.\mathrm{n}^{\circ} 26432\right)$, com curvas de nível apresentando equidistância de $20 \mathrm{~m}$, com auxílio de pontos cotados no terreno por GPS. A ferramenta TOPOGRID, do pacote de análises espaciais do ARCMAP 9.2, foi utilizada para obtenção do MDE. O mapa proveniente do ARCMAP 9.2 foi rasterizado, com células de 5 × $5 \mathrm{~m}$, e convertido para o formato ASCII para possibilitar a transferência para o PCRaster.

Os dados originais de uso atual do solo da bacia hidrográfica foram gerados com auxílio de SIG por Araújo (2006). Assim, o mapa de uso ocupacional do solo (Figura 1b) da bacia do Ribeirão Marcela, no formato vetorial, foi rasterizado em células de $5 \times 5 \mathrm{~m}$, convertido para o formato ASCII e, posteriormente, transformado para o tipo específico de mapa do PCRaster.

Para o modelo CN-SCS, é necessário ainda definir o tipo de solo, podendo este ser enquadrado em A, B, C ou D. Para as condições de solos tropicais, é recomendável a utilização da taxa de infiltração de água como critério para escolha dos grupos de solos, uma vez que a estrutura destes é o atributo com maior influência no seu comportamento hidrológico, fazendo com que solos com altos teores de argila apresentem alta capacidade de infiltração (Araújo, 2006). Neste trabalho, utilizou-se o critério recomendado por Pruski et al. (1997) (Quadro 2). Os dados para mapeamento foram levantados com infiltrômetro de Guelph, obedecendo a um grid de 240 x $240 \mathrm{~m}$, com refinamento em quatro subgrids de 60 x $60 \mathrm{~m}$, totalizando 190 pontos amostrados no espaço da bacia. A geração do mapa de infiltração foi desenvolvida com base na geoestatística, conforme Gomes et al. (2007), por meio do pacote GStat (Pebesma \& Wesseling, 1998) do PCRaster (Figura 1a). A condição de umidade antecedente do solo como função da chuva ocorrida nos cinco dias anteriores para cada evento simulado foi considerada de acordo com a seguinte situação
(Tucci, 2005): AMC I (0,0-35,0 mm), AMC II (35,0$52,5 \mathrm{~mm})$ e AMC III (> 52,5 mm).

No que se refere ao modelo Mishra-Singh Modificado (CN-MMS), são necessários ainda dados (de forma distribuída) de umidade de saturação e umidade inicial do solo. De forma semelhante à taxa de infiltração, foram coletadas, nos mesmos pontos de infiltração, amostras de solo para desenvolvimento de curvas características de umidade do solo retida às tensões de 0, 2, 6, 10, 33 e $100 \mathrm{kPa}$, com seus respectivos mapeamentos realizados com base na geoestatística, com o pacote GStat do PCRaster.

Os dados de precipitação pluvial, assim como os de escoamento (hidrograma), associados a essas precipitações foram obtidos por meio de uma estação climatológica automática e um linígrafo automático localizados na bacia hidrográfica, ambos trabalhando a cada 15 minutos. Tanto a precipitação pluvial quanto a vazão foram discretizadas em $n$ intervalos de 15 minutos, em que $n$ é o tempo total entre o início e o fim da chuva.

Em função do mapa de infiltração de água no solo foi possível gerar o mapa de grupo de solos. Este, combinado com o mapa de uso atual do solo, proporcionou a estruturação do mapa final do parâmetro $\mathrm{CN}$, caracterizando a relação solo-cobertura. O mapa de CN é de suma importância para simulação do escoamento superficial quando da utilização do modelo CN-SCS, visto que os diferentes valores de $\mathrm{CN}$, os quais variaram de 35 a 96 , demonstram a variabilidade espacial da relação solo-cobertura, apresentando diferentes potenciais de geração de escoamento superficial ao longo da bacia hidrográfica do Ribeirão Marcela.

No que se refere à calibração do modelo CN-MMS, adotou-se como critério considerar a umidade inicial da bacia da seguinte forma: eventos que ocorreram entre janeiro e fevereiro (tensões de $2 \mathrm{kPa}$, média entre 2 e $6 \mathrm{kPa}$ e $6 \mathrm{kPa}$ ), março e abril (tensões de $2 \mathrm{kPa}$, média entre 2 e $6 \mathrm{kPa}, 6 \mathrm{kPa}, 10 \mathrm{kPa}$ e $33 \mathrm{kPa}) \mathrm{e}$ maio, junho e julho (tensões de 10, 33, 100 e $1.500 \mathrm{kPa}$ ).

\section{Análise da exatidão dos modelos testados}

A comparação dos modelos foi realizada com base no erro de estimativa dos modelos em relação aos

Quadro 2. Taxas de infiltração de água no solo para os diferentes grupos de solos propostos no modelo CNSCS

\begin{tabular}{crl}
\hline Grupo de solo & Infiltração & Característica \\
\hline & $\mathrm{mm} \mathrm{h}^{-1}$ & \\
$\mathrm{~A}$ & $>190,0$ & Arenoso e profundo \\
$\mathrm{B}$ & $40,0-190,0$ & Arenoso e profundidade moderada \\
$\mathrm{C}$ & $3,0-40,0$ & Mais argiloso que o B e baixa permeabilidade \\
$\mathrm{D}$ & $<3,0$ & Argiloso, raso e muito impermeável \\
\hline
\end{tabular}

Fonte: Pruski et al. (1997). 
valores observados, extraídos do hidrograma monitorado, cuja equação é a seguinte:

$$
\mathrm{e}(\%)=\frac{\left(\mathrm{Q}_{\text {est }}-\mathrm{Q}_{\text {obs }}\right)}{\mathrm{Q}_{\mathrm{obs}}} \cdot 100
$$

Para análise da exatidão da simulação, bem como qualificação dos modelos, foi aplicada a Raiz do Erro Quadrado (REQ) como outro parâmetro estatístico, com o intuito de quantificar o erro decorrente do processo de simulação na estimativa das lâminas de escoamento superficial quando da utilização dos modelos CN-SCS e CN-MMS. Cabe salientar que, quanto menor o valor de REQ, melhor será o desempenho do modelo.

$$
\operatorname{REQ}(\mathrm{mm})=\sqrt{\left(\mathrm{Q}_{\mathrm{obs}}-\mathrm{Q}_{\text {est }}\right)^{2}}
$$

\section{RESULTADOS E DISCUSSÃO}

No quadro 3 podem-se comparar os valores simulados pelo modelo CN-SCS aos observados, considerando as respectivas condições de umidade antecedente aos eventos de chuva. Nos quadros 4 e 5 estão apresentados dados de simulação usando o modelo CN-MMS; no primeiro, considerou-se $1.000 \mathrm{~mm}$ a profundidade da camada de controle do solo e, no segundo, levou-se em consideração a variabilidade da profundidade em função do tipo de solo, constituindo-se em um parâmetro de calibração adicional. Analisando os resultados exibidos nesses três quadros, é possível observar que os dois modelos, de forma geral, possibilitaram simular as lâminas de escoamento superficial produzidas na bacia hidrográfica de forma satisfatória, com o modelo $\mathrm{CN}$ MMS mostrando melhor desempenho quando comparado ao CN-SCS. Verifica-se, em todas as situações, tendência de superestimativa do escoamento simulado em relação ao observado.

O modelo CN-SCS apresentou erros elevados em quatro eventos de chuva (eventos 7, 9, 13 e 14). Esses quatro eventos tiveram valores consideráveis de precipitação pluvial antecedente (condição AMC III, ou seja, solo úmido), acarretando superestimativa dos valores simulados do escoamento superficial, indicando que o modelo CN-SCS, para a condição AMC III, não mostrou calibração satisfatória. Isso pode ser justificado, de acordo com Mishra et al. (2006), porque o modelo CN-SCS é dividido em apenas três classes de umidade (AMC I, AMC II e AMC III), o que muitas vezes dificulta sua utilização sob determinadas condições hidrológicas antecedentes. Essa situação significa que o modelo considera, por exemplo, a mesma influência de uma P5 (precipitação pluvial dos últimos cinco dias) de $60 \mathrm{~mm}$ e outra de $100 \mathrm{~mm}$ na umidade antecedente do solo, e, fisicamente, há importante

Quadro 3. Lâminas de escoamento superficial observadas para os diferentes eventos de precipitação pluvial

\begin{tabular}{|c|c|c|c|c|c|}
\hline Evento & Data & ES & Modelo CN & $\operatorname{AMC}^{(2)}(\mathrm{P} 5)$ & Erro \\
\hline & & & $-\mathrm{mm}-$ & - & $\%$ \\
\hline 1 & $31 / 1 / 2004$ & 0,660 & 0,684 & I $(0,00)$ & 3,64 \\
\hline 2 & $8 / 2 / 2004$ & 0,104 & 0,125 & $\mathrm{I}(7,00)$ & 20,19 \\
\hline 3 & $10 / 2 / 2004$ & 0,209 & 0,184 & I $(13,00)$ & $-11,96$ \\
\hline 4 & $14 / 2 / 2004$ & 0,352 & 0,347 & I $(0,00)$ & $-1,42$ \\
\hline 5 & $20 / 2 / 2004$ & 0,095 & 0,116 & I $(11,00)$ & 22,11 \\
\hline 6 & $21 / 2 / 2004$ & 0,572 & 0,578 & I $(22,00)$ & 1,05 \\
\hline 7 & $23 / 2 / 2004$ & 0,411 & 1,964 & III $(62,00)$ & 377,86 \\
\hline 8 & $23 / 2 / 2004$ & 0,378 & 0,375 & III $(100,00)$ & $-0,79$ \\
\hline 9 & $24 / 2 / 2004$ & 0,414 & 15,934 & III $(106,00)$ & 3748,79 \\
\hline 10 & $19 / 3 / 2004$ & 0,454 & 0,467 & I $(4,40)$ & 2,86 \\
\hline 11 & $4 / 4 / 2004$ & 0,178 & 0,196 & I $(0,00)$ & 10,11 \\
\hline 12 & $4 / 4 / 2004$ & 0,629 & 0,611 & I $(11,20)$ & $-2,86$ \\
\hline 13 & $5 / 4 / 2004$ & 0,375 & 10,602 & III $(56,60)$ & 2727,2 \\
\hline 14 & $7 / 4 / 2004$ & 0,319 & 10,154 & III $(86,70)$ & 3083,1 \\
\hline 15 & $12 / 4 / 2004$ & 0,400 & 0,373 & I $(12,10)$ & $-6,75$ \\
\hline 16 & $26 / 5 / 2004$ & 0,12 & 0,115 & I $(0,00)$ & $-4,17$ \\
\hline 17 & $31 / 5 / 2004$ & 0,462 & 0,434 & $\mathrm{I}(0,60)$ & $-6,06$ \\
\hline 18 & $19 / 7 / 2004$ & 0,061 & 0,050 & $\mathrm{I}(0,00)$ & 18,03 \\
\hline
\end{tabular}
do ano hidrológico 2004-2005 e simuladas de acordo com o modelo Curva-Número (CN - SCS)

${ }^{(1)}$ Valores estimados de acordo com o modelo CN. ${ }^{(2)}$ Condição antecedente de umidade de acordo com a precipitação dos últimos cinco dias. 
Quadro 4. Lâminas de escoamento superficial observadas para os diferentes eventos de precipitação pluvial do ano hidrológico 2004-2005 e simuladas de acordo com o modelo CN-MMS, considerando uma profundidade da camada de solo de $1.000 \mathrm{~mm}$

\begin{tabular}{|c|c|c|c|c|c|c|c|c|c|}
\hline \multirow{2}{*}{ Data } & \multirow{2}{*}{ ES } & \multicolumn{8}{|c|}{ Modelo CN-MMS a diferentes umidades $(\mathrm{kPa})$, em $\mathbf{m m}^{(1)}$} \\
\hline & & Entre 0 e 2 & 2 & Entre 2 e 6 & 6 & 10 & 33 & 100 & Erro \\
\hline & $\mathrm{mm}$ & & & & & & & & $\%$ \\
\hline $31 / 1$ & 0,660 & 3,182 & 0,647 & 0,625 & 0,613 & - & - & - & $-1,97$ \\
\hline $8 / 2$ & 0,104 & 0,101 & 0,114 & 0,111 & 0,105 & - & - & - & 0,96 \\
\hline $10 / 2$ & 0,209 & 0,210 & 0,196 & 0,201 & 0,233 & - & - & - & 0,48 \\
\hline $14 / 2$ & 0,352 & 0,354 & 0,347 & 0,318 & 0,318 & - & - & - & 0,57 \\
\hline $20 / 2$ & 0,095 & 0,253 & 0,095 & 0,094 & 0,113 & - & - & - & 0,00 \\
\hline $21 / 2$ & 0,572 & 0,604 & 0,543 & 0,658 & 0,709 & - & - & - & $-5,07$ \\
\hline $23 / 2$ & 0,411 & 0,873 & 0,392 & 0,432 & 0,349 & - & - & - & 5,11 \\
\hline $23 / 2$ & 0,378 & 0,380 & 0,401 & 0,301 & 0,255 & - & - & - & 0,53 \\
\hline $24 / 2$ & 0,414 & 8,476 & 2,132 & 0,504 & 0,427 & - & - & - & 3,14 \\
\hline $19 / 3$ & 0,454 & 0,455 & 0,452 & 0,426 & 0,502 & 0,497 & 0,500 & - & 0,22 \\
\hline $4 / 4$ & 0,178 & 0,170 & 0,188 & 0,181 & 0,175 & 0,139 & 0,097 & - & $-1,69$ \\
\hline $4 / 4$ & 0,629 & 0,846 & 0,604 & 0,586 & 0,598 & 0,587 & 0,583 & - & $-3,97$ \\
\hline $5 / 4$ & 0,375 & 1,344 & 0,379 & 0,341 & 0,388 & 0,376 & 0,428 & - & 0,27 \\
\hline $7 / 4$ & 0,319 & 1,152 & 0,317 & 0,309 & 0,130 & 0,239 & 0,258 & - & $-0,63$ \\
\hline $12 / 4$ & 0,400 & 0,380 & 0,360 & 0,307 & 0,422 & 0,377 & 0,371 & - & $-5,00$ \\
\hline $26 / 5$ & 0,120 & - & - & - & & 0,097 & 0,068 & 0,060 & $-19,17$ \\
\hline $31 / 5$ & 0,462 & - & - & - & - & 0,469 & 0,349 & 0,503 & 1,52 \\
\hline $19 / 7$ & 0,061 & - & - & - & - & 0,073 & 0,087 & 0,065 & 6,56 \\
\hline
\end{tabular}

(1) Valores de escoamento superficial estimados pelo modelo CN-MMS para cada condição de umidade antecedente; valores em negrito correspondem ao menor erro produzido; ES: escoamento superficial observado.

Quadro 5. Lâminas de escoamento superficial observadas para os diferentes eventos de precipitação pluvial do ano hidrológico 2004-2005 e simuladas de acordo com o modelo CN-MMS, considerando a camada de solo como variável

\begin{tabular}{|c|c|c|c|c|c|c|c|c|c|}
\hline \multirow{2}{*}{ Data } & \multirow{2}{*}{ ES } & \multicolumn{2}{|c|}{ Modelo } & \multicolumn{6}{|c|}{ CN-MMS a diferentes umidades $(\mathrm{kPa})$, em $\mathrm{mm}^{(1)}$} \\
\hline & & Entre 0 e 2 & 2 & Entre 2 e 6 & 6 & 10 & 33 & 100 & Erro \\
\hline & $\mathrm{mm}$ & & & & & & & & $\%$ \\
\hline $31 / 1$ & 0,660 & 3,182 & 0,647 & 0,625 & 0,613 & - & - & - & $-1,97$ \\
\hline $8 / 2$ & 0,104 & 0,101 & 0,114 & 0,111 & 0,105 & - & - & - & 0,96 \\
\hline $10 / 2$ & 0,209 & 0,210 & 0,196 & 0,201 & 0,233 & - & - & - & 0,48 \\
\hline $14 / 2$ & 0,352 & 0,354 & 0,347 & 0,318 & 0,318 & - & - & - & 0,57 \\
\hline $20 / 2$ & 0,095 & 0,253 & 0,095 & 0,094 & 0,113 & - & - & - & 0,00 \\
\hline $21 / 2$ & 0,572 & 0,604 & 0,543 & 0,658 & 0,709 & - & - & - & $-5,07$ \\
\hline $23 / 2$ & 0,411 & 0,873 & 0,392 & 0,432 & 0,349 & - & - & - & 5,11 \\
\hline $23 / 2$ & 0,378 & 0,380 & 0,401 & 0,301 & 0,255 & - & - & - & 0,53 \\
\hline $24 / 2$ & 0,414 & 8,476 & 2,132 & 0,504 & 0,427 & - & - & - & 3,14 \\
\hline $19 / 3$ & 0,454 & 0,455 & 0,452 & 0,426 & 0,502 & 0,497 & 0,500 & - & 0,22 \\
\hline $4 / 4$ & 0,178 & 0,170 & 0,188 & 0,181 & 0,175 & 0,139 & 0,097 & - & $-1,69$ \\
\hline $4 / 4$ & 0,629 & 0,846 & 0,604 & 0,586 & 0,598 & 0,587 & 0,583 & - & $-3,97$ \\
\hline $5 / 4$ & 0,375 & 1,344 & 0,379 & 0,341 & 0,388 & 0,376 & 0,428 & - & 0,27 \\
\hline $7 / 4$ & 0,319 & 1,152 & 0,317 & 0,309 & 0,130 & 0,239 & 0,258 & - & $-0,63$ \\
\hline $12 / 4$ & 0,400 & 0,380 & 0,360 & 0,307 & 0,422 & 0,377 & 0,371 & - & $-5,00$ \\
\hline $26 / 5$ & 0,120 & - & - & - & - & 0,097 & 0,068 & 0,060 & $-19,17$ \\
\hline $31 / 5$ & 0,462 & - & - & - & - & 0,469 & 0,349 & 0,503 & 1,52 \\
\hline $19 / 7$ & 0,061 & - & - & - & - & 0,073 & 0,087 & 0,065 & 6,56 \\
\hline
\end{tabular}

(1) Valores de escoamento superficial estimados pelo modelo CN-MMS para cada condição de umidade antecedente; valores em negrito correspondem ao menor erro produzido. ES: escoamento superficial observado. 
diferença entre essas duas situações no tocante à geração de escoamento, dificultando um ajuste ideal do modelo. Segundo esses autores, este fato comprova a importância da incorporação do parâmetro diretamente associado à umidade antecedente para o cálculo da abstração inicial, como uma função tanto da precipitação pluvial antecedente quanto da retenção potencial máxima. Nesse mesmo aspecto, Mello et al. (2007) demonstraram, por meio de modelos estatísticos, a importância da umidade antecedente no comportamento geral do modelo CN-SCS tanto na estimativa das condições de abstração inicial quanto nos valores de $\mathrm{CN}$ propriamente ditos, influenciando diretamente o escoamento superficial direto.

Quanto ao modelo CN-MMS (Quadros 4 e 5), este gerou resultados simulados próximos aos observados, com valores de REQ baixos (Quadro 6), principalmente em condições de maior umidade do solo, sendo possível afirmar que, em condições mais secas, o modelo foi mais sensível no que diz respeito ao coeficiente de abstração inicial $(\lambda)$. Mishra et al. (2006) relatam que essa boa adaptação do modelo se dá em virtude da inserção da umidade antecedente no cálculo da abstração inicial como uma função da precipitação pluvial antecedente e da retenção potencial máxima, tornando o modelo mais próximo da realidade física da gênese do escoamento, quando comparado ao modelo CN-SCS.

Analisando ainda o quadro 6, podem-se comparar os resultados gerados pelo modelo CN-SCS aos obtidos pelo modelo CN-MMS, este último nas suas duas situações de análise. O modelo CN-SCS gerou resultados com maiores valores de REQ, de acordo com as estatísticas apresentadas (valores mínimo, máximo, média e mediana), quando comparado ao modelo $\mathrm{CN}$ -
MMS, podendo-se enquadrá-lo em uma categoria inferior. Por sua vez, o modelo CN-MMS apresentou valores de REQ muito baixos, especialmente os valores médio e máximo, demonstrando ser um modelo superior ao CN-SCS. Comparando o modelo CN-MMS com um único valor de profundidade com o mesmo modelo avaliado com profundidade variável da camada de solo, pode-se perceber que não existe diferença importante entre os dois, demonstrando que para a bacia hidrográfica do Ribeirão Marcela a característica profundidade da camada de solo não apresenta grande sensibilidade dentro do modelo. Isso pode ser explicado pela predominância dos Latossolos, os quais, segundo Araújo (2006), são profundos e com perfil homogêneo em termos de seus atributos físicos controladores da infiltração de água. Portanto, no contexto da geração do escoamento superficial, a camada de solo, nesse caso, não foi significativa.

Os valores de CV (Quadro 6) dizem respeito à dispersão ocorrida entre os valores de REQ para os 18 eventos de chuva-vazão aplicados ao processo de calibração. Pode-se notar que o método CN-SCS produziu maior CV quando comparado ao CN-MMS, e isso ocorreu em virtude da maior amplitude dos valores encontrados de REQ para o primeiro método, o qual apresentou maiores erros de estimativa, demonstrando sua qualidade inferior; é necessária a inclusão de outras variáveis de entrada, especialmente associadas à umidade antecedente do solo, para que haja melhora no seu desempenho.

No quadro 7 estão apresentados os intervalos de valores encontrados para o parâmetro $\lambda$, bem como seus valores de média e mediana, para cada modelo usado na simulação, permitindo analisar o comportamento desse parâmetro em função da

Quadro 6. Desempenho dos modelos de acordo com a estatística REQ

\begin{tabular}{|c|c|c|c|c|c|}
\hline Modelo & Mínimo & Máximo & Média & Mediana & CV \\
\hline & \multicolumn{4}{|c|}{$-\mathrm{mm}$} & $\%$ \\
\hline $\mathrm{CN}-\mathrm{SCS}$ & 0,003 & 15,520 & 2,076 & 0,021 & 223,881 \\
\hline CN-MMS, camada de solo de $1.000 \mathrm{~mm}$ & 0,000 & 0,029 & 0,008 & 0,003 & 118,269 \\
\hline CN-MMS, camada de solo variável & 0,000 & 0,023 & 0,006 & 0,003 & 123,748 \\
\hline
\end{tabular}

Quadro 7. Variação dos valores do coeficiente de abstração inicial ( $\lambda$ ) para os modelos aplicados

\begin{tabular}{|c|c|c|c|c|c|}
\hline Modelo & Mínima & Máxima & Média & Mediana & CV \\
\hline & \multicolumn{4}{|c|}{$-\mathrm{mm}$} & $\%$ \\
\hline CN-SCS & 0,0200 & 0,500 & 0,224 & 0,165 & 78,837 \\
\hline CN-MMS, camada de solo de $1.000 \mathrm{~mm}$ & 0,0001 & 0,500 & 0,133 & 0,060 & 119,351 \\
\hline CN-MMS, camada de solo variável & 0,0001 & 0,500 & 0,063 & 0,020 & 166,161 \\
\hline
\end{tabular}


umidade do solo antecedente. Mishra et al. (2003, 2006) sugerem calibrar valores entre 0 e 0,5 , embora haja possibilidade de ele ser superior a 0,5 . No modelo CN-SCS não foi possível obter resultados satisfatórios para todos os eventos com o intervalo de $\lambda$ sugerido, principalmente nos eventos de chuva com umidade do solo antecedente classificada como úmida (AMC III), gerando erros consideráveis. $O$ valor médio de $\lambda$ por este método foi de 0,224 com mediana de 0,165 , próximo ao sugerido pelo modelo $(\lambda=0,2)$, conforme Jain et al. (2006) e Mishra et al. (2003). A pequena divergência entre os valores de $\lambda$ padrão e os obtidos neste trabalho pode ser explicada, segundo Mishra et al. (2003), pela diferença entre os fatores meteorológicos, como temperatura e radiação solar, que interferem no cálculo da evapotranspiração, bem como pelo regime pluvial da região, alterando as condições de umidade antecedente e, consequentemente, os valores de $\lambda$. Os coeficientes de variação (Quadro 7) estão relacionados à variabilidade encontrada entre os valores calibrados do parâmetro $\lambda$, para cada um dos modelos. A menor variabilidade dos valores calibrados de $\lambda$, para o modelo CN-SCS, é justificada pela existência de apenas três condições de umidade antecedente, as quais são caracterizadas por intervalos de precipitação pluvial antecedente, conforme comentado anteriormente, $e$ não pela umidade propriamente dita, reduzindo a sensibilidade do modelo quanto à calibração de $\lambda$. De forma oposta, o modelo CN-MMS apresentou maior variabilidade dos valores em razão de o cálculo destes ser baseado diretamente nos valores de umidade do solo, demonstrando, uma vez mais, a alta sensibilidade do parâmetro à umidade do solo antecedente aos eventos de precipitação.

Por outro lado, apesar de sua maior variabilidade, conseguiu-se calibrar o parâmetro $\lambda$ de forma adequada para o modelo CN-MMS, tanto com camada de solo fixada em $1.000 \mathrm{~mm}$ quanto com profundidade variável; na primeira situação, os valores de média e mediana foram de 0,136 e 0,06 e, na segunda, de 0,063 e 0,020 , respectivamente. Os valores calibrados neste trabalho também diferem daquele tomado como referência, conforme discutido anteriormente, porém são semelhantes aos obtidos em trabalhos realizados por Mishra et al. (2006) e Mello et al. (2008), sendo o primeiro aplicando o modelo CN-MMS com dados de diversas bacias hidrográficas rurais norte-americanas, com área entre 0,17 e 71,99 ha, tendo os autores encontrado um ajuste considerado bom para $\lambda$, em que este parâmetro oscilou entre 0 e 0,21 . No trabalho de Mello et al. (2008), realizado em uma bacia hidrográfica predominantemente rural com área de $2.080 \mathrm{~km}^{2}$ no Brasil e usando o mesmo modelo para estimativa da lâmina de escoamento superficial num modelo de simulação hidrológica, encontraram-se valores de $\lambda$ entre 0,01 e 0,2. Dessa forma, não é possível fixar um valor para $\lambda$ sem considerar as condições antecedentes de umidade do solo, sendo este um parâmetro altamente sensível e sua análise, com base nas condições de umidade antecedente do solo, imprescindível para um desempenho satisfatório dos modelos, concordando com observações e conclusões obtidas por trabalhos anteriores que trataram desse assunto (Mishra et al., 2006; Mello et al., 2007).

\section{CONCLUSÕES}

1. Foi possível observar que as lâminas simuladas pelos modelos estruturados no SIG PCRaster apresentaram resultados satisfatórios, demonstrando que a aplicação deles em ambiente SIG produz melhores resultados. O modelo CN-MMS promoveu melhor desempenho, comprovando a sensibilidade do parâmetro Curva-Número à umidade antecedente aos eventos de precipitação pluvial, sendo fundamental sua incorporação ao processo. No entanto, o modelo CN-SCS, sob condições de solo muito úmido (AMC III), superestimou o escoamento superficial na bacia hidrográfica, mostrando que é necessário algum cuidado na sua utilização para esta condição de umidade antecedente do solo, mesmo numa condição de ambiente SIG.

2. O modelo CN-MMS mostrou maior facilidade na calibração dos valores de $\lambda$, parâmetro este que está associado à abstração inicial da chuva, demonstrando pequena variabilidade no tocante à camada de solo para as condições da bacia hidrográfica estudada; no entanto, é essencial a incorporação da umidade antecedente do solo na sua calibração.

\section{LITERATURA CITADA}

ARAÚJO, A.R. Solos da Bacia do Alto Rio Grande (MG): Base para estudos hidrológicos e aptidão agrícola. Lavras, Universidade Federal de Lavras, 2006. 345p. (Tese de Doutorado)

BACIC, I.L.; ROSSITER, D.G. \& MANNAERTS, C.M Applicability of a distributed watershed pollution model in a data-poor environment in Santa Catarina State, Brazil. R. Bras. Ci. Solo, 32:1699-1712, 2008.

DE ROO, A.P.J. \& JETTEN, V.G. Calibrating and validating the LISEM model for two data sets from the Netherlands and South Africa. Catena, 37:477-493, 1999.

GOMES, N.M.; SILVA, A.M.; MELLO, C.R. \& FARIA, M.A. Métodos de ajuste e modelos de semivariograma aplicados ao estudo da variabilidade espacial de atributos físicohídricos do solo. R. Bras. Ci. Solo, 31:435-443, 2007.

HRISSANTHOU, V. Comparative application of two erosion models to a basin. Hydrol. Sci. J., 47:279-292, 2002.

JAIN, M.K.; MISHRA, S.K. \& SINGH, V.P. Evaluation of AMCdependent SCS-CN-based models using watershed characteristics. Water Res. Manag., 20:531-552, 2006 
JETTEN, V. PCRaster - Manual do usuário. Utrecht, Centre for Environment and Landscape Dynamics, University of Utrecht, 2003. 100p.

MELLO, C.R.; VIOLA, M.R.; NORTON, L.D.; SILVA, A.M. \& WEIMAR, F.A. Development and application of a simple hydrologic model simulation for a Brazilian headwater basin. Catena, 75:235-247, 2008.

MELLO, C.R.; LIMA, J.M. \& SILVA, A.M. Simulação do deflúvio e vazão de pico em microbacia hidrográfica com escoamento efêmero. R. Bras. Eng. Agric. Amb., 11:410419, 2007.

MISHRA, S.K.; SAHU, R.K.; ELDHO, T.I. \& JAIN, M.K. An improved Ia-S relation incorporating antecedent moisture in SCS-CN methodology. Water Res. Manag., 20:643-660, 2006.

MISHRA, S.K.; SINGH, V.P.; SANSALONE, J.J. \& ARAVAMUTHAN, V. A modified SCS-CN method: Characterization and testing. Water Res. Manag., 17:3768, 2003.

NOTTER, B.; MACMILLAN, L.; VIVIROLI, D.; WEINGARTNER, R. \& LINIGER, H.P. Impacts of environmental change on water resources in the Mt. Kenya region. J. Hydrol., 343:266-278, 2007.

PEBESMA, E.J. \& WESSELING, C.G. Gstat: A program for geostatistical modelling, prediction and simulation. Comp. Geosci., 24:17-31, 1998 .
PEDROSA, B.M. \& CÂMARA, G. Modelagem dinâmica e sistemas de informações geográficas. In: MEIRELLES, M.S.P.; CÂMARA, G. \& ALMEIDA, C.M. Geomática: Modelos e aplicações ambientais. Brasília, Embrapa, 2007. p.235-280

PRUSKI, F.F.; FERREIRA, P.A.; RAMOS, M.M. \& CECON, P.R. A model to design level terraces. J. Irrig. Drain. Eng., 123:8-12, 1997.

SOIL CONSERVATION SERVICE. Hydrology; National Engineering Handbook. Washington, Soil Conservation Service/USDA, 1971. Supplement A, Section 4.

TUCCI, C.E.M. Modelos hidrológicos. 2.ed. Porto Alegre, Universidade Federal do Rio Grande do Sul, 2005. 678p.

van DEURSEN, W.P.A. Geographical Information Systems and Dynamic Models: Development and application of a prototype spatial modelling language. Utrecht, University of Utrecht, 1995. 180p. (Tese de Doutorado)

von STACKELBERG, N.O.; CHESCHEIR, G.M.; SKAGGS, R.W. \& AMATYA, D.M. Simulation of the hydrologic effects of aforestation in the Tacuarembó River Basin, Uruguay. Trans. ASABE, 50:455-468, 2007.

WESSELING, C.G.; KARSSENBERG, D.J.; BURROUGH, P.A. \& van DEURSEN, W.P.A. Integrated dynamic environmental models in GIS: The development of a dynamic modelling language. Trans. GIS, 11:40-48, 1996. 